\section{EFETIVIDADE DE WEBSITE DE EDUCAÇÃO EM SAÚDE BUCAL PARA ADOLESCENTES}

\author{
Effectiveness of an educational website on oral health for \\ adolescents
}

\author{
La efectividad de website de educación en la salud bucal de \\ adolescentes
}

\section{RESUMO}

Objetivo: Avaliar a efetividade de um website desenvolvido como ferramenta de educação em saúde bucal para adolescentes. Métodos: Estudo experimental realizado no município de Belém-PA, no período entre agosto e novembro de 2012, com amostra composta por 160 adolescentes na faixa etária de 11 a 15 anos, de escola pública e particular. Formaramse dois grupos, sendo um experimental com participação em atividade educativa e acesso a um website de informações sobre saúde bucal para adolescentes; e o controle com palestras contendo a mesma temática. Os dados foram coletados por meio de questionários autoadministrados e, depois da ação educativa, foram verificados a partir de Teste Quiquadrado, Análise de Variância e Teste de Tukey. Resultados: Observou-se que os escolares, de ambos os grupos, após as atividades educativas, obtiveram um bom conhecimento acerca das questões sobre cárie e etiologia do câncer bucal, tendo os escolares do grupo experimental apresentado melhor desempenho sobre esses temas. A maioria $(n=122$; $76,25 \%$ ) dos escolares participantes da pesquisa nunca havia realizado o autoexame bucal e não possuía conhecimento adequado sobre doença periodontal. Conclusão: Os dois métodos educativos fizeram com que houvesse uma mudança nos conhecimentos adquiridos pelos adolescentes, o que se reflete na quantidade de respostas acertadas por eles. Porém, como pode ser constatado, o grupo experimental obteve melhor desempenho após o acesso ao website, mostrando sua real efetividade.

Descritores: Educação em Saúde Bucal; Saúde do Adolescente; Hebiatria.

\section{ABSTRACT}

Objective: To evaluate the effectiveness of a website developed as an educational tool on oral health for adolescents. Methods: Experimental study developed in the city of Belem, Para, in the period between August and November 2012, with sample consisting of 160 adolescents ranging from 11 to 15 years old, attending public or private schools. Two groups were formed, the intervention one, with participation in educational activity and access to a website with information on oral health for adolescents; and the control group, with talks on the same topic. Data was collected using two self-administered questionnaires and, after the educational intervention, were assessed using Chi-square test, Analysis of Variance and Tukey's test. Results: After the educational activities, students from both groups presented good knowledge about issues related to dental caries and etiology of oral cancer, having the experimental group presented a better performance on these topics. Most of the students $(n=122 ; 76.25 \%)$ participants in this survey had never held the oral self-examination and did not have adequate knowledge about periodontal disease. Conclusion: Both educational method led to a change in the knowledge acquired by the adolescents, which was reflected in the number of correct answers they gave. As it can be seen, however, the intervention group attained better performance after access to the website, showing its effectiveness.

Descriptors: Dental Health Education; Adolescent Health; Adolescent Medicine.
Artigo Original
1) Centro Universitário do Estado do Pará CESUPA - Belém (PA) - Brasil
Recebido em: 28/01/2014 Revisado em: 15/03/2014 Aceito em: 18/05/2014 


\section{RESUMEN}

Objetivo: Evaluar la efectividad de un website desarrollado como herramienta de educación en salud bucal de adolescentes. Métodos: Estudio experimental realizado en el municipio de Belém-PA, entre agosto y noviembre de 2012, con una muestra de 160 adolescentes entre los 11 y 15 años, de escuela pública y privada. Dos grupos, siendo uno experimental con participación en actividad educativa y acceso a um website de informaciones sobre la salud bucal de adolescentes; y el grupo control con ponencias de la misma temática. Fueron recogidos datos a través de cuestionarios auto-administrados tras la acción educativa. Los mismos fueron analizados con la prueba del Chi-cuadrado, Análisis de la Varianza y la Prueba de Tukey. Resultados: Se observó que los escolares de ambos los grupos tenían un buen conocimiento sobre caries y etiología del cáncer bucal tras las actividades educativas, siendo el grupo experimental el que presento mejor desempeño sobre estos temas. La mayoría $(n=122$; $76,25 \%$ ) de los escolares participantes de la investigación nunca habian realizado el autoexamen bucal y no tenían el conocimiento adecuado sobre enfermedad periodontal. Conclusión: Los dos métodos educativos generaron cambios en los conocimientos adquiridos de los adolescentes lo que se refleja en la cantidad de respuestas correctas de su parte. Sin embargo, como se pudo ver, el grupo experimental tuvo mejor desempeño tras el acceso al website, señalando su real efectividad.

Descriptores: Educación en Salud Dental; Salud del Adolescente; Medicina del Adolescente.

\section{INTRODUÇÃO}

A adolescência, do latim adolesço, que significa crescer, desenvolver-se ${ }^{(1)}$, é uma fase específica do desenvolvimento humano, caracterizada por um período de mudanças e transformações múltiplas, profundas e fundamentais para que o ser humano possa atingir a maturidade e inserir-se na sociedade no papel de adulto ${ }^{(2)}$. Essa etapa é de grande valia, pois, nela, os aprendizados adquiridos não são esquecidos. Segundo a Organização Mundial de Saúde (OMS), a adolescência é uma fase compreendida entre 10 e $19 \operatorname{anos}^{(3)}$; já a legislação brasileira e o Estatuto da Criança e do Adolescente (ECA) consideram adolescentes os indivíduos compreendidos entre 12 e 18 anos de idade ${ }^{(4)}$.

A adolescência é uma fase de estruturação da personalidade e de definição da identidade ${ }^{(5)}$. Na busca de um equilíbrio físico, psicológico e social, o adolescente apresenta comportamentos extremos, ora exacerbando suas atitudes positivas, ora se mostrando francamente negligente com seus cuidados à saúde. Não raro, a adolescência é tida como um período de risco aumentado à cárie dentária, em decorrência do precário controle de biofilme dentário e redução dos cuidados com a escovação dentária ${ }^{(6)}$. Esse ciclo de vida se apresenta como um período de risco para a saúde bucal ${ }^{(7)}$. Usa-se o termo "odontohebiatria" para especificar o profissional da odontologia capacitado em atender adolescentes ${ }^{(8)}$.

A saúde bucal de um adolescente pode ser um bom indicador da sua saúde geral. A educação em saúde tornase um dos pilares da odontologia moderna na busca pela integralidade de suas ações ${ }^{(9)}$. A inclusão dos adolescentes em programas preventivos direcionados a eles, junto com uma abordagem cuidadosa, criteriosa e sensível por parte do profissional, pode contribuir para que cheguem à vida adulta de maneira saudável ${ }^{(10)}$.

Indubitavelmente, a promoção de saúde bucal tornase uma estratégia importante ao combate das doenças orais. Os adolescentes constituem a população-alvo para a implantação de medidas educativas, entre outras razões, por apresentarem características e atitudes singulares, e necessidades igualmente distintas. Em virtude da carência de metodologias de educação em saúde bucal destinadas ao público adolescente, ressalta-se a importância de métodos educativos que sejam desenvolvidos em ambientes de interesse dele, sendo, atualmente, o principal deles o computador $^{(11)}$.

Cerca de 56 milhões de pessoas acessam a internet no Brasil, e a maior parcela desse grupo é composta por jovens entre 10 e 19 anos $^{(12)}$. A tendência desses acessos é aumentar, já que os jovens estão sendo criados em um mundo cada vez mais conectado, sendo possível, então, lançar mão dessa tendência para ações educativas ${ }^{(13)}$.

Baseado nos fatores citados e na necessidade da implantação de metodologias educativas efetivas e específicas para o público adolescente, este estudo tem como objetivo avaliar a efetividade de um website desenvolvido como ferramenta de educação em saúde bucal para adolescentes.

\section{MÉTODOS}

Estudo transversal, de caráter exploratório, com amostra de conveniência e coleta de dados envolvendo adolescentes do ensino médio de escolas do município de Belém-PA durante o período letivo de 2012.

A população do presente estudo foi composta por 198 adolescentes, sendo selecionados 160 , na faixa etária de 11 a 15 anos, devidamente matriculados nas escolas e de ambos os sexos, sendo estes os critérios de inclusão. Excluíramse aqueles que não manifestaram interesse em participar do estudo e os que não responderam aos questionários de forma completa.

Os pesquisadores, antes do início da pesquisa, foram calibrados sobre a metodologia utilizada e a coleta de dados. Além disso, houve aplicação do questionário utilizado como 
instrumento de avaliação a 10 adolescentes, como estudo piloto, com o objetivo de identificar possíveis dúvidas e verificar se o tempo para responder ao questionário e para a realização das outras atividades era suficiente.

Duas escolas, uma da rede pública e outra da rede privada, com sistema de inclusão digital e um espaço adequado para a realização das atividades cumpriram os requisitos dos critérios de seleção. Por meio de ofício dirigido à direção das escolas, solicitou-se a autorização para a realização da presente pesquisa e, após sua obtenção, pôde ser realizado o contato com os alunos das escolas, informando-os sobre o caráter, objetivo e importância da pesquisa, convidando-os a participar dela. Os adolescentes e seus responsáveis assinaram o Termo de Consentimento Livre e Esclarecido, totalizando 160 questionários válidos, sendo 80 de cada escola.

Investigou-se o website Odontologia Plugada, destinado a fornecer informações sobre saúde bucal ao adolescente, mostrando e ensinando a importância desta no cotidiano, estimulando o desenvolvimento de hábitos bucais saudáveis e o autocuidado, possuindo uma ferramenta para portadores de deficiência.

Em cada escola, os adolescentes foram divididos em dois grupos de 40: 1) Grupo teste: com acesso ao website educativo Odontologia Plugada, disponível em <http:// www.odontologiaplugada.com.br $>$. Continha assuntos de interesse, como os relacionados à doença da gengiva, cárie dental, odontologia estética, alimentação saudável, traumatismo dental e câncer bucal. O tempo de acesso ao site compreendeu 40 minutos. 2) Grupo controle: palestra educativa interativa abordando os mesmos temas contidos no website, utilizando slides, cartazes ilustrativos e macromodelos, com tempo de duração de 40 minutos.

O instrumento de avaliação utilizado foi um questionário contendo 12 questões objetivas e subjetivas relacionadas aos temas abordados em ambas as metodologias educativas, aplicado em dois momentos: um inicial, para avaliar o conhecimento prévio dos escolares e um segundo momento, após a metodologia aplicada (website ou palestra), com a finalidade de avaliar o grau de conhecimento adquirido por eles ao final das atividades.

Os dados apurados no Programa BioEstat 5.0. elaboraram tabelas contendo informações sobre as medidas de frequências nas variáveis categóricas e de média e desvio padrão das variáveis de acerto das questões. Utilizou-se o Teste do Qui-quadrado para verificar a associação entre as variáveis categóricas, a Análise de Variância (ANOVA) a um critério e o Teste de Tukey para verificação dos grupos em que havia diferença significante entre os dados sobre acertos das questões. Para a tomada de decisão nas análises, adotou-se o nível de significância $(\alpha)$ de 0,05 .
O projeto foi previamente submetido e aprovado pelo Comitê de Ética em Pesquisa (CEP) - CESUPA, sob $n^{\circ}$ 113.908 e Certificado de Apresentação para Apreciação Ética (CAAE) $n^{\circ}$ 05164512.7.0000.5169.

\section{RESULTADOS}

Na Tabela I, estão apresentados os conhecimentos e as práticas de saúde bucal adquiridos pelos adolescentes do grupo controle e do grupo experimental após a realização das atividades educativas. No que diz respeito ao conceito e conhecimento de cárie, os adolescentes de ambos os grupos obtiveram um bom desempenho: 50 (62,5\%) do grupo controle e $61(76,2 \%)$ do grupo experimental. Estatisticamente, não houve diferença entre os grupos $(\mathrm{p}=0,0592)$, porém, ressalta-se que o grupo experimental teve um melhor desempenho numérico.

Com relação ao conhecimento sobre as causas da cárie, $67(83,7 \%)$ adolescentes do grupo controle e $76(95 \%)$ do grupo experimental responderam adequadamente à questão, mas os do grupo experimental também tiveram um melhor desempenho $(\mathrm{p}=0,0209)$.

Quanto à transmissibilidade de bactérias causadoras da cárie, $69(86,2 \%)$ dos adolescentes do grupo controle responderam corretamente a questão, tendo este grupo apresentado um desempenho mais eficaz quando comparado ao grupo experimental, em que $50(62,5 \%)$ responderam adequadamente $(\mathrm{p}=0,0006)$.

A respeito da conduta que se deve ter diante de uma lesão de cárie, tanto os adolescentes do grupo controle $(\mathrm{n}=70 ; 87,5 \%)$ quanto os do grupo experimental $(\mathrm{n}=73$; $91,2 \%)$, responderam adequadamente, não apresentando diferenças significantes $(\mathrm{p}=0,4415)$.

No que concerne ao conhecimento sobre câncer bucal, apenas $14(17,5 \%)$ dos adolescentes do grupo controle e $30(37,5 \%)$ dos adolescentes do grupo experimental responderam corretamente, evidenciando diferença entre os grupos $(\mathrm{p}=0,0046)$. Apesar do conhecimento relativamente baixo e de não saberem descrever o que é o câncer bucal, os adolescentes do grupo controle $(n=51 ; 63,7 \%)$ e do grupo experimental $(n=75 ; 93,7 \%)$ demonstraram ter um conhecimento pertinente acerca da etiologia dessa doença, entretanto, o grupo experimental teve uma resposta mais satisfatória $(\mathrm{p}<0,0001)$.

Com relação à realização do autoexame bucal, 20 (25\%) dos escolares do grupo controle e 18 (22,5\%) dos escolares do grupo experimental relataram não ter realizado ainda esse exame, não havendo diferença entre os grupos $(\mathrm{p}=0,7102)$.

Sobre a conduta que se deve ter diante de situações de traumatismo dentário, seja qual for a causa, os adolescentes 
do grupo controle $(\mathrm{n}=68 ; 85 \%)$ tiveram um desempenho melhor quando comparados aos do grupo experimental $(\mathrm{n}=63 ; 78,7 \%)$, com ambos respondendo adequadamente $(\mathrm{p}=0,3048)$. Porém, no que concerne às medidas de prevenção de traumatismo dentário, durante a realização de práticas esportivas, apenas 18 (22,5\%) dos adolescentes do grupo controle relataram ter um conhecimento pertinente e adequado acerca das condutas preventivas, enquanto no grupo experimental, do total, $39(48,8 \%)$ responderam corretamente. Apesar de o grupo experimental ter apresentado melhor desempenho em relação ao conhecimento sobre traumatismo dentário $(\mathrm{p}=0,0005)$, notou-se que a maioria dos escolares, de ambos os grupos, não tem um conhecimento adequado sobre que conduta deve-se tomar diante da situação que lhes foi exposta.

Dos adolescentes participantes da pesquisa, 72 (90\%) do grupo controle e $78(97,5 \%)$ do grupo experimental relataram ter um conhecimento adequado sobre alimentação saudável. Estatisticamente, não houve diferença entre os grupos $(\mathrm{p}=0,1025)$, porém, observa-se que o grupo

Tabela I - Conhecimentos e práticas de saúde bucal após atividades educativas entre adolescentes dos grupos controle e experimental em frequências absolutas e percentuais. Belém-PA, 2013.

\begin{tabular}{|c|c|c|c|c|c|}
\hline \multirow{2}{*}{ Conhecimentos e práticas } & \multicolumn{2}{|c|}{ Grupo Controle } & \multicolumn{2}{|c|}{ Grupo Experimental } & \multirow{2}{*}{$p^{*}$} \\
\hline & $\mathbf{n}$ & $\%$ & $\mathbf{n}$ & $\%$ & \\
\hline \multicolumn{6}{|l|}{ Conceito de cárie } \\
\hline Adequado & 50 & 62,5 & 61 & 76,2 & 0,0592 \\
\hline Inadequado & 30 & 37,5 & 19 & 23,8 & \\
\hline \multicolumn{6}{|l|}{ Causas da cárie } \\
\hline Adequado & 67 & 83,7 & 76 & 95,0 & 0,0209 \\
\hline Inadequado & 13 & 16,3 & 4 & 5,0 & \\
\hline \multicolumn{6}{|l|}{ Transmissibilidade de bactérias causadoras da cárie } \\
\hline Adequado & 69 & 86,2 & 50 & 62,5 & 0,0006 \\
\hline Inadequado & 11 & 13,8 & 30 & 37,5 & \\
\hline \multicolumn{6}{|l|}{ Conduta diante de lesão de cárie } \\
\hline Adequada & 70 & 87,5 & 73 & 91,2 & 0,4415 \\
\hline Inadequada & 10 & 12,5 & 7 & 8,8 & \\
\hline \multicolumn{6}{|l|}{ Conhecimento sobre câncer bucal } \\
\hline Adequado & 14 & 17,5 & 30 & 37,5 & 0,0046 \\
\hline Inadequado & 66 & 82,5 & 50 & 62,5 & \\
\hline \multicolumn{6}{|l|}{ Conhecimento sobre etiologia do câncer bucal } \\
\hline Adequado & 51 & 63,7 & 75 & 93,7 & $<0,0001$ \\
\hline Inadequado & 29 & 36,3 & 5 & 6,3 & \\
\hline \multicolumn{6}{|l|}{ Realização de autoexame bucal } \\
\hline Fez & 20 & 25,0 & 18 & 22,5 & 0,7102 \\
\hline Não fez & 60 & 75,0 & 62 & 77,5 & \\
\hline \multicolumn{6}{|l|}{ Conhecimento sobre alimentação saudável } \\
\hline Adequado & 72 & 90,0 & 78 & 97,5 & 0,1025 \\
\hline Inadequado & 8 & 10,0 & 2 & 2,5 & \\
\hline \multicolumn{6}{|l|}{ Conduta diante de traumatismo dentário } \\
\hline Adequada & 68 & 85,0 & 63 & 78,7 & 0,3048 \\
\hline Inadequada & 12 & 15,0 & 17 & 21,3 & \\
\hline \multicolumn{6}{|l|}{$\begin{array}{l}\text { Conduta preventiva de traumatismo dentário em } \\
\text { práticas esportivas }\end{array}$} \\
\hline Adequada & 18 & 22,5 & 39 & 48,8 & 0,0005 \\
\hline Inadequada & 62 & 77,5 & 41 & 51,2 & \\
\hline \multicolumn{6}{|l|}{ Conhecimento sobre sangramento gengival } \\
\hline Adequado & 66 & 82,5 & 69 & 86,2 & 0,5136 \\
\hline Inadequado & 14 & 17,5 & 11 & 13,8 & \\
\hline \multicolumn{6}{|l|}{ Conhecimento sobre doença periodontal } \\
\hline Adequado & 21 & 26,3 & 37 & 46,3 & 0,0085 \\
\hline Inadequado & 59 & 73,7 & 43 & 53,7 & \\
\hline Total & 80 & 100,0 & 80 & 100,0 & -------- \\
\hline
\end{tabular}


experimental teve um desempenho relativamente maior que o grupo controle, apresentando um número maior de alunos que responderam adequadamente.

A respeito do conhecimento que os adolescentes adquiriram sobre sangramento gengival, 66 (82,5\%) do grupo controle e $69(86,2 \%)$ do grupo experimental relataram que não é normal a gengiva sangrar, não apresentando diferença estatisticamente significante entre os grupos $(\mathrm{p}=0,5136)$. Porém, os do grupo experimental tiveram um desempenho mais eficaz.

Apesar de os adolescentes de ambos os grupos relatarem que o sangramento gengival em condições de saúde do periodonto não é normal, apenas $21(26,3 \%)$ do grupo controle e 37 (46,3\%) do grupo experimental mostraram ter um conhecimento adequado e pertinente acerca da doença periodontal e o que se deve fazer para evitá-la. Não obstante a maioria dos escolares ter respondido inadequadamente a essa questão, pode-se observar que os do grupo experimental tiveram um desempenho melhor ( $\mathrm{p}=0,0085)$.

$\mathrm{Na}$ Tabela II, focaliza-se a quantidade de questões que os adolescentes de ambos os grupos acertaram em momentos distintos, ou seja, antes e após a realização das atividades educativas sobre saúde bucal. Observa-se que, no grupo controle, a média de acertos por aluno foi de 5,8 questões antes da realização da palestra e, no grupo experimental, a média foi de 6,5 questões, antes do acesso ao website, o que permite constatar que os grupos eram estatisticamente semelhantes nesse momento inicial. Após a execução das atividades educativas, o grupo controle obteve uma média de acertos de 7,3 questões, enquanto no grupo experimental, a média foi de 8,4 questões, demonstrando que este grupo obteve melhor desempenho que o grupo controle $(\mathrm{p}<0,05)$.

$\mathrm{Na}$ Tabela III, visualiza-se a quantidade de respostas adequadas sobre conhecimentos e práticas acerca da saúde bucal, após a realização das atividades educativas, entre os estudantes dos grupos controle e experimental de escola pública e privada. Avaliando-se os escolares pertencentes à escola pública, nota-se que os do grupo controle obtiveram um pior desempenho que os do grupo experimental $(\mathrm{p}<0,05)$, com média de acertos de 6,1 e 7,8 questões, respectivamente. Analisando-se os adolescentes pertencentes à escola privada, observa-se que, após a palestra, a média de acertos no grupo controle foi de 8,6 questões e, no grupo experimental, de 9,0 questões.

Tabela II - Medidas de respostas adequadas sobre conhecimentos e práticas de saúde bucal entre adolescentes dos grupos controle e experimental nos momentos antes e após a realização de atividades educativas. Belém-PA, 2013.

\begin{tabular}{lcccc}
\hline \multirow{2}{*}{ Medidas } & \multicolumn{2}{c}{ Grupo Controle } & \multicolumn{2}{c}{ Grupo Experimental } \\
\cline { 2 - 5 } & Antes & Depois & Antes & Depois \\
\hline Amostra & 80 & 80 & 80 & 80 \\
Média & $5,8^{\mathrm{A}}$ & $7,3^{\mathrm{B}}$ & $6,5^{\mathrm{A}}$ & $8,4^{\mathrm{C}}$ \\
Desvio padrão & 1,7 & 2,2 & 1,4 & 1,7 \\
\hline $\mathrm{p}<0,05$ (Análise de Variância). Letras diferentes nos valores da média representam diferenças estatisticamente significantes (p<0,05 \\
no Teste de Tukey).
\end{tabular}

Tabela III - Medidas de respostas adequadas sobre conhecimentos e práticas de saúde bucal entre adolescentes dos grupos controle e experimental de escolas públicas e privadas após a realização de atividades educativas. Belém-PA, 2013.

\begin{tabular}{lcccc}
\hline \multirow{2}{*}{ Medidas } & \multicolumn{2}{c}{ Grupo Controle } & \multicolumn{2}{c}{ Grupo Experimental } \\
\cline { 2 - 5 } & Escola Pública & Escola Privada & Escola Pública & Escola Privada \\
\hline Amostra & 40 & 40 & 40 & 40 \\
Média & $6,1^{\mathrm{A}}$ & $8,6^{\mathrm{BC}}$ & $7,8^{\mathrm{B}}$ & $9,0^{\mathrm{C}}$ \\
Desvio padrão & 1,8 & 1,9 & 1,7 & 1,4 \\
\hline p $<0,05$ (Análise de Variância). & Letras diferentes nos valores da média representam diferenças estatisticamente significantes (p<0,05 \\
no Teste de Tukey). & \multicolumn{4}{c}{}
\end{tabular}

\section{DISCUSSÃO}

A educação em saúde representa uma estratégia fundamental no processo de formação de comportamentos que promovam e mantenham a saúde ${ }^{(14)}$, devendo ser efetiva a ponto de melhorar o conhecimento dos indivíduos. A escola representa um ambiente social e educacional favorável para se trabalhar conhecimentos e mudanças de comportamento.

O alerta para a compreensão dos adolescentes e suas necessidades é de suma importância, principalmente para que os profissionais de saúde ou educação se esforcem na tentativa de influenciar positivamente essa parcela da população, que pode atuar como agentes multiplicadores 
de saúde ${ }^{(15)}$. A revolução tecnológica abriu caminho para mudanças no processo de ensino e aprendizagem, sendo a internet um instrumento pedagógico disponível e de grande influência, graças à popularização de seu uso e riqueza de conteúdo ${ }^{(16)}$.

Com relação às metodologias educativas utilizadas, os adolescentes que participaram do presente estudo se mostraram interessados, observadores, críticos e participativos quanto aos temas abordados nas atividades aplicadas. Assim como em um estudo realizado em Vitória de Santo Antão- $\mathrm{PE}^{(15)}$, que avaliou, através de formulário semiestruturado, adolescentes com idade entre 10 e 16 anos, de escola pública e particular, sobre a importância da saúde bucal, cuja maioria dos adolescentes demonstrou domínio e conhecimento a respeito da cárie, citando-a como a doença bucal mais conhecida.

Os dados encontrados na presente pesquisa divergem de um estudo realizado no município de Araçatuba-SP(17), que avaliou, por meio da técnica do grupo focal e de questionários, o entendimento de adolescentes em relação à saúde bucal, revelando que mais da metade dos adolescentes que participaram $(56,67 \%)$ alegou desconhecimento sobre a transmissibilidade da cárie e suas bactérias, visto que a palavra "transmissão" não havia sido compreendida, em um primeiro momento, pelos adolescentes.

Uma condição indispensável no processo educativo em saúde bucal é ouvir e entender o paciente ${ }^{(18)}$ e/ou o indivíduo que está recebendo orientações. A partir do momento em que estes interpretam corretamente a palavra, o entendimento e a participação deles acerca do tema tende a ser maior. O uso de uma linguagem técnica torna-se corriqueiro na vida dos cirurgiões-dentistas, a ponto de não perceberem que esse tipo de vocabulário foge à compreensão das pessoas leigas ${ }^{(19)}$.

Mesmo a cárie sendo a doença bucal mais enfocada pela mídia e por campanhas educativo-preventivas de uma forma geral ${ }^{(20)}$, e os adolescentes terem apresentado um conhecimento adequado sobre essa doença na investigação em questão, a adolescência é, por si só, um período de exacerbação da atividade de cárie, em virtude das mudanças físicas, psicológicas e sociais inerentes ao período, aumentando a incidência de lesões cariosas ${ }^{(21)}$.

Observou-se que o câncer bucal é um assunto pouco difundido entre os adolescentes participantes do atual estudo, visto que eles não possuíam tanto conhecimento a respeito do tema abordado, o que está de acordo com um estudo realizado em Campina Grande- $\mathrm{PB}^{(22)}$, que avaliou, por meio de um questionário, o conhecimento de adolescentes entre 10 e 19 anos de idade sobre saúde bucal, relatando que 436 (83\%) dos adolescentes informaram não ter conhecimento sobre o câncer de boca, evidenciando que esse assunto não é muito difundido. Durante a realização das atividades educativas na presente pesquisa, os adolescentes relataram que o tema despertou-lhes bastante interesse, aumentando, dessa maneira, a participação deles na discussão. Sendo assim, fizeram muitas perguntas, explanaram dúvidas acerca do tema discutido e situações que presenciaram ou ouviram falar, durante alguma fase de suas vidas, no meio onde vivem. A carência e/ou falta de uma divulgação pertinente sobre câncer bucal talvez seja a responsável pelo baixo conhecimento desses adolescentes sobre o tema. Dessa maneira, fica evidente a necessidade de uma educação em saúde que inclua estratégias preventivas e educativas em relação ao tema abordado.

Assim como a mídia e os outros meios de comunicação têm influência e destaque na divulgação de informações sobre saúde bucal, o cirurgião-dentista tem um papel de suma importância na educação em saúde, evidenciado em um estudo realizado em Duque de Caxias-RJ ${ }^{(14)}$, que avaliou, através de questionários, adolescentes entre 13 e 19 anos de idade, abordando questões relativas à saúde bucal e à consulta ao cirurgião-dentista, cuja principal fonte de informação sobre saúde bucal citada foi o cirurgião-dentista.

O profissional que lida com o paciente hebiatra deve incentivá-lo a adotar uma alimentação balanceada, tendo em vista que, além da cárie dental, a obesidade também é uma das doenças nutricionais que mais têm aumentado nos últimos anos e está relacionada, em geral, com o sedentarismo e os hábitos alimentares inadequados, os quais podem levar a sérios distúrbios na saúde geral, além de alterações bucais ${ }^{(10)}$. Abordar o paciente adolescente sobre a dieta não é uma tarefa fácil. As orientações quanto ao horário e à frequência de ingestão dos alimentos, assim como sobre sua consistência, também devem ser reforçadas, de modo a permitir um consumo inteligente de açúcar ${ }^{(21)}$.

Os resultados do presente estudo permitem inferir que o sangramento gengival não foi encarado como uma situação comum pelos adolescentes, mas como um problema de saúde bucal que merecesse tratamento. Em contrapartida, há estudos ${ }^{(14,17)}$ que revelam que há adolescentes que consideram normal o sangramento gengival, não associando essa alteração a algum tipo de doença. $\mathrm{O}$ conhecimento relativamente baixo dos adolescentes acerca da doença periodontal conota a necessidade de tal problema de saúde bucal ser abordado de maneira mais expressiva e significante.

O estilo de vida e a inconsequência, próprios da adolescência, fazem com que, durante essa fase, os indivíduos estejam mais suscetíveis a acidentes de diferentes naturezas ${ }^{(21)}$.

Durante as atividades educativas realizadas, a utilização de protetores bucais na prevenção dos traumas 
foi a conduta preventiva mais citada pelos adolescentes, pois, de acordo com eles, ajudaria a distribuir o impacto e, consequentemente, protegeria os dentes e estruturas adjacentes.

O critério socioeconômico utilizado no atual estudo foi o tipo de escola, e ele mostrou-se relevante no conhecimento prévio e no obtido após as intervenções educativas, influenciando na quantidade de questões acertadas.

Assim como na pesquisa em questão, estudos nacionais ${ }^{(15,23)}$ que avaliaram, através de questionário, o conhecimento sobre saúde bucal de adolescentes entre 10 e 16 anos de idade também evidenciaram que essa variável socioeconômica influencia em aspectos relacionados a conhecimentos e práticas sobre saúde bucal. A falta de informações pertinentes e de condutas adequadas acerca desses assuntos e as dificuldades de acesso a serviços de saúde por parte da população mais necessitada refletem nessa diferença.

A motivação e a educação em saúde bucal são ferramentas poderosas que, quando empregadas de maneira agradável, atrativa, cativante e divertida na transmissão de informações, apresentam-se eficazes, sendo esse o principal objetivo do website avaliado, desenvolvido de maneira moderna do ponto de vista estético, abordando, na linguagem dos adolescentes, de forma prazerosa e consistente, vários temas relacionados à saúde bucal, com imagens, textos, sons e jogos. Como o presente estudo revelou, o website demonstrou-se parcialmente efetivo em determinados assuntos. Os áudios que continham informações acerca desses assuntos demoravam a carregar, apresentando falhas durante a reprodução, sendo esse problema mais evidenciado nas escolas públicas, em virtude da velocidade da rede. O direcionamento e apoio ao paciente hebiatra contribuem para a formação de uma geração saudável, no conceito mais amplo da palavra, uma vez que a saúde bucal reflete a saúde geral do indivíduo.

\section{CONCLUSÃO}

Os resultados do presente estudo permitem concluir que os dois métodos educativos fizeram com que houvesse uma mudança nos conhecimentos adquiridos pelos adolescentes, o que se reflete na quantidade de respostas acertadas por eles. Porém, como pôde ser constatado, o grupo experimental obteve melhor desempenho após o acesso ao website, mostrando a real efetividade deste.

\section{REFERÊNCIAS}

1. Grossman E. A construção do conceito de adolescência no Ocidente. Adolesc Saúde, 2010;7(3):47-51.
2. Carvalho RWF. Aspectos psicossociais dos adolescentes de Aracaju (SE) relacionados à percepção de saúde bucal. Ciênc Saúde Coletiva. 2011;16(Supl 1):1621-8.

3. Ministério da Saúde (BR), Secretaria de Atenção à Saúde, Secretaria de Vigilância em Saúde. SB Brasil 2010: Pesquisa Nacional de Saúde Bucal: resultados principais. Brasília: Ministério da Saúde; 2012.

4. Brasil. Estatuto da Criança e do Adolescente: lei n. ${ }^{\circ}$ 8.069 de 13 de julho de $1990.1 .^{\circ}$ ed. Salvador: Egba; 2005.

5. Araújo EDS, Blankb N, Ramos JH. Comportamentos de risco à saúde de adolescentes do ensino médio. Rev Bras Promoç Saúde. 2009;22(3):164-71.

6. Gibilini C, Esmeriz CEC, Volpato LF, Meneghim ZMAP, Silva DD, Sousa MLR. Acesso a serviços odontológicos e auto-percepção da saúde bucal em adolescentes, adultos e idosos. Arq Odontol. 2010;46(4):213-23.

7. Garbin CAS, Garbin AJI, Moimaz SAS, Gonçalves PE. A saúde na percepção do adolescente. Physis. 2009; 19 (1):227-38 .

8. Toassi R, Ceriotti F. Prevalência e severidade de cárie dental e necessidades de tratamento em uma população de baixo nível socioeconômico de uma escola pública da área urbana de Lages, Santa Catarina (Brasil). Rev ABO Nac. 2010;18(Supl 1):70-4.

9. Cruz DI, Paulo RRD, Dias WS, Martins VF, Gandolf PE. O uso das mídias digitais na educação em saúde. Cad FUCAMP. 2011;10(13):130-42.

10. Saiani RAS, Queiroz AM, Raffaini MEG, BagatinRossi CR. Odontohebiatria: uma nova especialidade na odontologia. Rev Odontol UNICID. 2008;20(1):60-5.

11. Turioni APS, Salomão FGD, Monti JFC, Vasques FL, Cortelazz KL, Pereira AC. Avaliação das ações de educação em saúde bucal de adolescentes dentro da estratégia saúde da família. Ciênc Saúde Coletiva. 2012;17(7):841-8.

12. Instituto Brasileiro de Geografia e Estatística - IBGE. Sala de imprensa. Notícia [acesso em 02 Fev 2012]. Disponível em: http://saladeimprensa.ibge.gov.br/notic ias? view $=$ noticia $\&$ id $=1 \&$ busca $=1 \&$ idnoticia $=1517$

13. Storolli EAF, Bressan GC, Moreira RPG, Utino VM. Internet e adolescência: pontos positivos [trabalho de conclusão de curso]. São Paulo: Universidade de São Paulo; 2011.

14. Silva RT, Freixinho ABS, Miasato JM. Verificação do conhecimento e hábitos de saúde bucal em adolescentes 
de uma escola particular. Rev Odontol Univ Cid São Paulo. 2012;2 (1):19-25.

15. Granville-Garcia AF, Cavalcanti AL, Lorena Sobrinho JE, Araújo JC, Menezes VA, Barbosa AMF. Importância da saúde bucal: um enfoque em adolescentes de Vitória de Santo Antão-PE. Cad Saúde Colet (Rio J.). 2009;17(2):361-74.

16. Silva ACCG, Oliveira AAP, Ferreira Júnior M. Webliografia básica em promoção de saúde e prevenção de doenças. Rev Bras Promoç Saúde. 2009;22(4): 217-24.

17. Francisco KMS, Sundefeld MLMM, Garbin AJI, Garbin CAS. Técnica do grupo focal como método de avaliação do conhecimento de adolescentes sobre saúde bucal. Acta Sci Health Sci. 2009;31(2):165-70.

18. Ferraz CA, Barros CS, Vieira LB. Utilização de instrumentos para avaliar ações educativas em saúde bucal: um relato de experiência universitária. Rev Baiana Saúde Pública. 2009;33(2):58-67.

19. Zhu L, Petersen PE. Oral health knowledge, attitudes and behaviour of children and adolescents in China. Int Dent J. 2003;53(5):289-98.

20. Granville-Garcia AF, Sobrinho JEL, Menezes VA, Cavalcanti AL. Ocorrência de tabagismo e fatores associados em escolares. RFO UPF. 2008;13(1):30-4.
21. Guedes-Pinto AC, Bonecker M, Rodrigues CRMD. Fundamentos de Odontologia: Odontopediatria. $1^{\mathrm{a}}$ ed. In: Ferreira FM, Rodrigues CRMD, editores. Atendimento Odontológico a adolescentes. São Paulo: Livraria Santos Editora; 2009. p. 381-407.

22. Granville-Garcia AF, Fernandes LV, Farias TSS, Bento PM, Medeiros CLSG, Menezes VA. Importância da Saúde Bucal entre Adolescentes de Escolas Públicas de Campina Grande/PB, Brasil. Pesq Bras Odontoped Clin Integr. 2011;11(3):425-31.

23. Granville-Garcia AF, Sobrinho JEL, Araujo JC, Menezes VA, Costa EMMB. Influência do Fator Socioeconômico no Comportamento dos Adolescentes em Relação à Saúde Bucal. Odonto (São Bernardo do Campo). 2008;16(31):53-61.

\section{Endereço para correspondência:}

Priscilla Bittencourt de Almeida Figueiredo Centro Universitário do Estado do Pará (CESUPA)

Av. Governador José Malcher, 1963

Bairro: Nazaré

CEP: 66060-230 - Belém - PA - Brasil

E-mail: priscilla@cesupa.br 